01;07

\title{
Взаимная синхронизация наногенераторов, связанных с помощью спиновых волн
}

\author{
() К.Н. Алешин, В.В. Матросов, К.Г. Мишагин
}

Нижегородский государственный университет им. Н.И. Лобачевского, Нижний Новгород, Россия

E-mail: kirill_al@bk.ru

Поступило в Редакцию 26 июля 2017 г.

Рассмотрены динамические режимы системы двух наногенераторов, связанных через спиновые волны, в зависимости от силы связи и частотной расстройки между ними. Получено разбиение пространства параметров системы на области существования различных динамических режимов: синхронного, квазисинхронного, биений и подавления колебаний генераторов. Выделены области мультистабильного поведения. Исследовано поведение на границах областей захвата и удержания синхронного режима, приведены зависимости ширины полос захвата и удержания от силы связи между наногенераторами.

DOI: 10.21883/PJTF.2018.07.45878.16985

Прохождение постоянного электрического тока через многослойную металлическую структуру, состоящую из чередующихся ферромагнитных и немагнитных слоев, может приводить к генерации СВЧколебаний в широком диапазоне частот [1]. На основе подобных структур реализуются спиновые наногенераторы, практическое применение которых в настоящее время проблематично из-за высокого уровня фазовых шумов и малой мощности. Взаимная синхронизация нескольких наногенераторов позволяет устранить указанные недостатки 
и приводит к подавлению шумов и когерентному сложению мощностей генерируемых сигналов.

Ранее в [2] рассмотрены вопросы синхронизации колебаний наногенератора внешним сигналом. Настоящая работа продолжает начатые исследования, в ней рассматриваются вопросы взаимной синхронизации двух наногенераторов, каждый из которых представляет собой точечный контакт, расположенный на общем проводящем слое гетерогенной структуры. При пропускании постоянного электрического тока через оба контакта в одном из слоев, называемом свободным, происходит возбуждение стоячей автоволны. Так как частота генерации зависит от плотности электрического тока, геометрии слоев и точечного контакта, спиновая волна будет образована двумя модами с собственными частотами $\omega_{1}$ и $\omega_{2}$.

Динамика каждой моды описывается уравнением для комплексной амплитуды моды [3]

$$
\begin{aligned}
& \frac{d c_{1}}{d t}=-j \omega_{1} c_{1}-\left(\eta_{1}-\beta_{1} J_{1}\right) c_{1}-j T_{1} \alpha_{1} n_{1} c_{1}-\frac{3 \beta_{1} J_{1}}{2 S N}\left(u_{1}^{2}+v_{1}^{2}\right) n_{1} c_{1} \\
& -j \frac{2}{3} T_{1} \lambda^{2}\left(\delta_{1} n_{2} c_{1}+\delta c_{1}^{*} c_{2} c_{2}\right)-\frac{\beta_{1} J_{1}}{S N} \lambda^{2}\left[2\left(u_{2}^{2}+v_{2}^{2}\right) n_{2} c_{1}+u_{2}^{2} c_{1}^{*} c_{2} c_{2}\right]
\end{aligned}
$$

где $c_{1}$ - комплексная амплитуда моды спиновой волны, возбуждаемой первым наногенератором, $\omega_{1}-$ ее собственная частота, $\eta_{1}-$ скорость релаксации, $\beta_{1}$ - параметр, характеризующий эффект переноса спина, $J_{1}$ - плотность электрического тока, $\lambda-$ параметр связи в интервале $[0,1], j$ - мнимая единица. Значение и смысл остальных параметров приведены в [3]. Уравнение для второй моды получается взаимной заменой индексов 1 и 2 .

Система (1) определена в четырехмерном фазовом пространстве $U=\left\{\operatorname{Re} c_{1}, \operatorname{Im} c_{1}, \operatorname{Re} c_{2}, \operatorname{Im} c_{2}\right\}$. Анализ фазовых траекторий в пространстве $U$ выявил возможность существования аттракторов двух типов: предельные циклы и инвариантные торы. Если компоненты $c_{1}$ и $c_{2}$ не обращаются в нуль, то предельный цикл является образом синхронного режима, когда мгновенные частоты мод равны; инвариантный тор соответствует режиму биений, когда мгновенные частоты мод не равны. Если действительная и мнимая компоненты комплексной амплитуды $c_{i}$ стационарного процесса равны нулю, то такой режим

Письма в ЖТФ, 2018, том 44, вып. 7 
будем называть режимом подавления моды $i$-го генератора. Изучение предельных циклов и инвариантных торов в фазовом пространстве $U$ является довольно сложной задачей. Исследование динамики спиновых волн можно существенно упростить, если для описания спиновых волн использовать фазовые уравнения, которые получаются из уравнений (1) с помощью замены $c_{i}=\rho_{i} \exp \left(j \varphi_{i}\right)$, где $\rho_{i}$ - действительная амплитуда, а $\varphi_{i}-$ фаза первой моды спиновой волны. Переходя к разности фаз $\theta=\varphi_{2}-\varphi_{1}$, получаем систему фазовых уравнений, эквивалентную системе (1):

$$
\left\{\begin{aligned}
\frac{d \rho_{1}}{d \tau}= & -\left(a_{2}+a_{3} \rho_{1}^{2}\right) \rho_{1}-\lambda^{2} \rho_{2}^{2} \rho_{1}\left(a_{21}-a_{12} \sin \theta+a_{22} \cos \theta\right) \\
\frac{d \rho_{2}}{d \tau}=- & \left(b_{2}+b_{3} \rho_{2}^{2}\right) \rho_{2}-\lambda^{2} \rho_{1}^{2} \rho_{2}\left(b_{21}+b_{12} \sin \theta+b_{22} \cos \theta\right) \\
\frac{1}{2} \frac{d \theta}{d \tau}= & \left(-\gamma-b_{1} \rho_{2}^{2}+a_{1} \rho_{1}^{2}\right)-\lambda^{2} \rho_{1}^{2}\left(b_{11}+b_{12} \cos \theta-b_{22} \sin \theta\right) \\
& +\lambda^{2} \rho_{2}^{2}\left(a_{11}+a_{12} \cos \theta-a_{22} \sin \theta\right)
\end{aligned}\right.
$$

Здесь $\tau=t \omega_{1}-$ безразмерное время, $\theta=\varphi_{2}-\varphi_{1}-$ мгновенная разность фаз колебаний двух мод, $\gamma=\left(\omega_{2} / \omega_{1}-1\right)$ - относительная частотная расстройка, параметры $a_{1}=-1.0147, a_{2}=-0.0372$, $a_{3}=0.4751, a_{11}=-1.3570, a_{12}=-0.6777, a_{21}=0.6119, a_{22}=0.2087$, $b_{1}=-1.022, b_{2}=-0.0447, b_{3}=0.4983, b_{11}=-1.5636, b_{12}=0.4347$, $b_{21}=0.6757, b_{22}=0.2285$ вычислены на основе физических параметров натурного эксперимента [3].

Модель (2) определена в цилиндрическом фазовом пространстве $U_{1}=\left\{\theta(\bmod 2 \pi), \rho_{1}, \rho_{2}\right\}$, где режиму синхронизации соответствует состояние равновесия, режиму биений - вращательный предельный цикл (цикл, охватывающий фазовый цилиндр $U_{1}$ ). Кроме того, в фазовом пространстве $U_{1}$ могут существовать колебательные предельные циклы, которые являются образом квазисинхронного режима, в котором частоты мод равны в среднем за период колебаний. Заметим, что в фазовом пространстве $U_{1}$ квазисинхронному режиму, как и режиму биений, отвечает инвариантный тор. Разделение инвариантных торов на образы квазисинхронного режима и режима биений - крайне затратная вычислительная процедура, связанная с анализом периодов квазипериодических решений на торе.

Письма в ЖТФ, 2018, том 44, вып. 7 
Отметим, что переход к фазовым уравнениям для изучения синхронизации связанных наногенераторов с помощью спиновых волн применялся, например, в [4], однако в этой работе использована модель линейной связи мод в отличие от модели, рассматриваемой здесь, в которой взаимодействие мод учитывается нелинейным образом как результат четырехволнового взаимодействия магнонов.

На рис. 1, а представлено разбиение плоскости параметров модели (2) на области существования режимов: $D_{s}-$ синхронизации, $D_{k}-$ квазисинхронизации, $D_{b}-$ биений, $D_{10}$ и $D_{01}$ - подавления мод первого и второго генераторов. Существует три области синхронизации: левая, правая и центральная.

Центральная область $D_{s}$ примыкает к точке $N_{0}(0.0138763,0)$ и является наиболее крупной. $\mathrm{C}$ увеличением $\lambda$ полоса синхронизации сначала расширяется, достигает максимума на уровне $\lambda=0.6$, далее сужается. Эту область ограничивают сплошные линии бифуркационных кривых: двукратного равновесия (расположенные ниже точек $N_{1}$ и $N_{3}$ ), Андронова-Хопфа (между точками $N_{1}, N_{2}$ и $N_{3}, N_{4}$ ), трехкратного равновесия (участки кривых выше точек $N_{2}$ и $N_{4}$ ) [5]. Отметим, что бифуркация Андронова-Хопфа является жесткой; следовательно, соответствующие ей участки границ области синхронизации являются опасными [6]. При выходе из области $D_{s}$ через правую границу ниже точки $N_{2}$ и через левую границу ниже точки $N_{4}$ устанавливается режим биений. Границы области захвата в синхронный режим показаны штриховыми линиями, области совместного существования синхронного режима и режима биений отмечены штриховкой. Выход из области синхронизации вправо выше точки $N_{2}$ и влево выше $N_{4}$ связан с подавлением мод второго и первого генераторов соответственно. Области биений и подавления колебаний разделяют штрихпунктирные бифуркационные прямые, проходящие через точки $N_{2}$ (0.0539385, $0.815932)$ и $N_{4}(-0.0369919,0.919174)$, соответствующие касательной бифуркации вращательных предельных циклов.

Структуры левой и правой областей синхронизации, примыкающих к ним областей квазисинхронизации и областей биений аналогичны. Фрагмент плоскости параметров с левой областью синхронизации приведен на рис. $1, b$. Здесь области расходятся из точки $N_{5}$ $(-0.0322808,0.815932)$, при этом крайняя левая область - область синхронизации, средняя - квазисинхронизации, правая - биений. Левой границей области $D_{s}$ синхронизации служит бифуркационная 

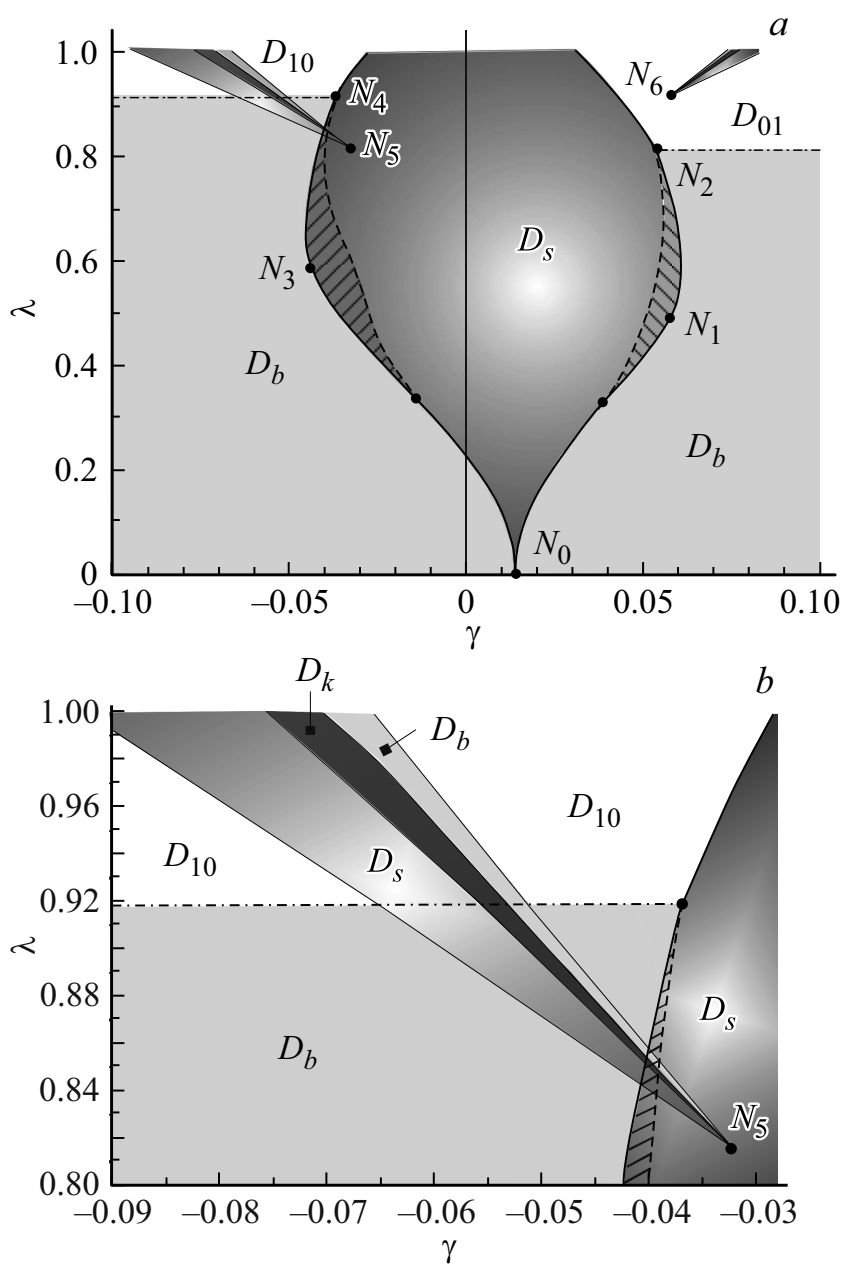

Рис. 1. Структура $(a)$ и фрагмент $(b)$ плоскости параметров модели (2). $D_{s}-$ область синхронизации, $D_{k}-$ область квазисинхронизации, $D_{b}-$ область биений, $D_{10}$ и $D_{01}-$ подавления мод первого и второго генераторов.

Письма в ЖТФ, 2018, том 44, вып. 7 


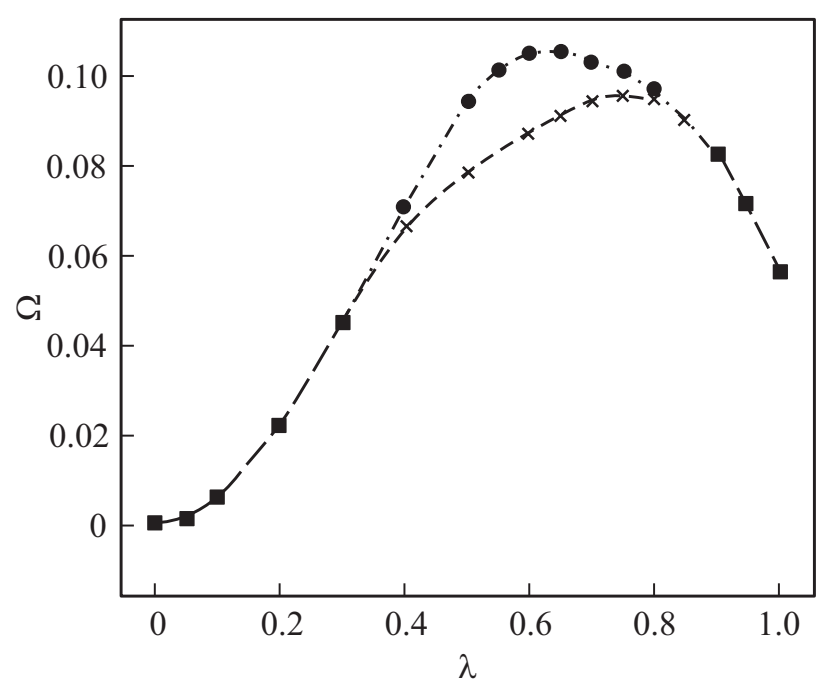

Рис. 2. Зависимость ширины полос захвата и удержания от величины параметра связи $\lambda$, рассчитанная по центральной области $D_{s}$. Штриховая линия - ширина полосы захвата, штрихпунктирная - ширина полосы удержания.

кривая трехкратного состояния равновесия, области $D_{s}$ и $D_{k}$ разделяет кривая мягкой бифуркации Андронова-Хопфа, области $D_{k}$ и $D_{b}-$ кривая гомоклинических траекторий. Примечательно, что точка $N_{5}$ принадлежит центральной области синхронизации, поэтому области $D_{s}, D_{k}$ и $D_{b}$ пересекаются с центральной областью синхронизации, порождая большое разнообразие картин мультистабильного поведения, в частности одновременное существование двух синхронных режимов, синхронного и квазисинхронного режимов, двух режимов биений и синхронного режимов.

Области $D_{s}, D_{k}$ и $D_{b}$ правой структуры расходятся из точки $N_{6}(0.0576322,0.919174)$, при этом область $D_{s}$ расположена справа, $D_{b}$ - слева, $D_{k}$ - в центре. Точка $N_{6}$ не принадлежит центральной области, поэтому зоны мультистабильного поведения правой структурой не порождаются.

Используя данные о расположении границ областей бистабильности и синхронизации, можно оценить зависимость ширины полос захва-

Письма в ЖТФ, 2018, том 44, вып. 7 
та и удержания от величины параметра связи $\lambda$. Эта зависимость представлена на рис. 2. Ширина полосы $\Omega$ вычисляется как разность максимальных и минимальных значений частотной расстройки $\gamma$ на соответствующих границах областей при фиксированном значении параметра связи. На основании зависимости $\Omega(\lambda)$ можно вычислить значение параметра связи, при котором ширина полосы захвата будет максимальной: $\lambda_{\max }=0.75$.

В работе рассмотрены вопросы взаимной синхронизации двух спиновых наногенераторов, связанных через спиновые волны. Исследование проведено путем качественно-численного анализа динамической модели, определенной в цилиндрическом фазовом пространстве. Установлено соответствие между фазовыми траекториями модели и динамическими режимами связанных генераторов: синхронным, квазисинхронным, биений и подавления колебаний генераторов. Получено разбиение пространства параметров модели на области существования, различающиеся динамическим поведением, выделены области мультистабильного поведения. Исследовано поведение на границах областей захвата и удержания синхронного режима, приведены зависимости ширины полос захвата и удержания от силы связи между наногенераторами. В отличие от модели синхронизации внешним источником спиновых волн [2] обнаружены области существования квазисинхронного режима. Кроме того, появление взаимной связи приводит к возникновению побочных областей синхронизации внутри области биений.

\section{Список литературы}

[1] Slonczewski J.C. // J. Magn. Magn. Mater. 1996. V. 159. N 1. P. L1-L7.

[2] Алешин К.Н., Матросов В.В., Мишагин К.Г // Письма в ЖТФ. 2017. Т. 43. B. 6. C. $8-14$.

[3] Rezende S.M., de Aguiar F.M., Rodriguez-Suarez R.L., Azevedo A. // Phys. Rev. Lett. 2007. V. 98. N 8. P. 087202.

[4] Safin A.R., Udalov N.N., Kapranov M.V. // Eur. Phys. J. Appl. Phys. 2014. V. 67. N 2. P. 20601.

[5] Анищенко В.С. Сложные колебания в простых системах. М.: Наука, 1990. $312 \mathrm{c}$.

[6] Баутин Н.Н. Поведение динамических систем вблизи границ области устойчивости. М.: Наука, 1984. 176 с.

Письма в ЖТФ, 2018, том 44, вып. 7 